\title{
Paper on Designing Costless THz Paper Optics
}

\author{
A. Siemion, ${ }^{1}$ P. Kostrowiecki-Lopata, ${ }^{1}$ A. Pindur, ${ }^{1}$ P. Zagrajek, ${ }^{2}$ and M. Sypek ${ }^{1}$ \\ ${ }^{1}$ Faculty of Physics, Warsaw University of Technology, 75 Koszykowa Str., 00-662 Warsaw, Poland \\ ${ }^{2}$ Institute of Optoelectronics, Military University of Technology, 2 Kaliski Str., 00-908 Warsaw, Poland \\ Correspondence should be addressed to A. Siemion; agnieszka@if.pw.edu.pl
}

Received 8 August 2016; Accepted 7 November 2016

Academic Editor: Eric Freysz

Copyright (C) 2016 A. Siemion et al. This is an open access article distributed under the Creative Commons Attribution License, which permits unrestricted use, distribution, and reproduction in any medium, provided the original work is properly cited.

\begin{abstract}
Designing diffractive optical elements is crucial for efficient development of THz techniques. Here, we consider paper structures and we analyze their advantages and disadvantages in fast prototyping. The discussion about using material parameters like refractive index and absorption coefficient in designing diffractive optical elements is shown. We analyze the influence of phase step mismatch, of attenuation of real structure, and of nonuniform illumination on the efficiency of the structure. All these features result in worsening of the diffraction efficiency but they do not seem to have such significant influence as shadow effect introduced by fast varying zones. Diffractive elements can be designed with very good accordance with experimental results which makes them ideal for possible applications. Paper optics scan be used more for fast prototyping; nevertheless its performance can be increased by placing it inside water protecting foil.
\end{abstract}

\section{Introduction}

More and more efficient emission of $\mathrm{THz}$ radiation is opening new possibilities for security [1], noninvasive testing [2], material identification [3], and medical diagnosis [4]. THz waves are promising in detecting metal objects hidden behind something like it takes place in case of brown-up or white-up during landing of helicopters. To efficiently use and transform the $\mathrm{THz}$ radiation we can use beam shaping to form the desired intensity pattern and elements gathering and focusing the beam to significantly enhance the amount of radiation impinging the detector's surface and assuring larger signal and therefore more efficient detection. Moreover, such optics can significantly reduce optical cross-talk in the case of matrix of detectors.

Mirrors used for $\mathrm{THz}$ beams can reflect the radiation and are wavelength independent but positioning of the system is not easy and elements are rather expensive, especially when they have more complicated shapes. For diffractive elements, various structures complicated in shape are available (they can be easily manufactured with different 3D printing techniques, laser cutting, or even with milling but only for axially symmetrical structures). Such elements strongly depend on the design wavelength, but this drawback can be suppressed by clever designing [5].

\section{Materials for THz}

Because of the fast development of $\mathrm{THz}$ technology, it is crucial to find new materials which will allow for easy manufacturing of optical elements. The question is what material should we use to manufacture diffractive objects for $\mathrm{THz}$ waves. The perfect material should be inexpensive and easy in processing and should possess good optical parameters. In order to provide strong signal, attenuation coefficient should be low to prevent the structure from absorbing most of the incoming energy. Refractive index should be more or less constant in whole used $\mathrm{THz}$ region. There are many materials fulfilling these requirements. The most frequently used materials for fabricating $\mathrm{THz}$ components are Teflon, TPX [6], Zeonex [7], TOPAS [8] polytetrafluoroethylene (PE) [9], and high-density polyethylene (HDPE) [10]. Another material is high-resistivity silicon [11]; however more materials can be used in manufacturing $\mathrm{THz}$ beam shaping objects, for example, conductive polymers [12], metal [13, 14], natural stone [15], and metamaterials [16]. It should be noticed that 
more and more $3 \mathrm{D}$ printed materials, like polyamide 12 or other plastics $[17,18]$, are used to manufacture diffractive optical elements. Another attempt considers using paper for $\mathrm{THz}$ beam shaping purposes $[19,20]$.

The best solution is to find the material having the refractive index for particular design frequency in the 1.4-1.7 range. It results from the fact that for higher difference of refractive index between air and used material (e.g., air-silicone interface introduces the change of around 2.4, while air-paper around 0.5) we will encounter high Fresnel losses and therefore using some antireflection coatings is recommended. Nevertheless, in case of frequently used hemispherical lenses it is almost impossible to create good and efficient antireflection coating due to the fact that the light illuminates the lens from various angles. In case of too small refractive index the structure is becoming thick which significantly increases attenuation and independently shadow effect [21-23].

It should be also noticed that not all materials are ready for outdoor use. In this case paper structures are rather for fast prototyping; however, we can use protecting foils to protect them from humidity or rain.

\section{Designing DOEs}

The functioning of diffractive optical elements can be described by analogy to the diffractive grating. In this kind of structures, a part of the incident light is not changed and propagates in the same way after the object, but some of the energy is redirected and forms diffraction orders. On the contrary to refractive lenses, which collects almost $100 \%$ of the incident light in the focal spot (not taking into account their attenuation), their diffractive counterparts do not have $100 \%$ efficiency directed into one particular order. In case of diffraction gratings, depending on the structure, we can obtain different efficiency in different orders by manipulating grating parameters such as fill factor or thickness/transparency of the structure. Diffractive optical elements can introduce either changes of amplitude or phase. The former will attenuate the incident radiation, while the latter may have theoretically even up to $100 \%$ of efficiency (with continuous phase profile). Considering the binary diffraction grating the fill factor $a$ can be easily defined as the fraction of the period of the diffraction grating that is filled with the grating material (either changing amplitude or phase). However, for more complicated structures, the fill factor is not as clear in interpretation due to the fact that the shape, the transparency, the height of the structure, or the width of the designed zones are variously changing.

Therefore, it is easy to define the diffraction efficiency for binary phase grating in general case, which can be calculated using formulas (1) [24] as follows:

$$
\begin{aligned}
& \eta_{0}=1+4 a\left[\sin ^{2}\left(\frac{\phi}{2}\right)\right], \\
& \eta_{m}=\frac{4 \sin ^{2}(\phi / 2)}{(\pi m)^{2}} \sin ^{2}(\pi m a),
\end{aligned}
$$

where $m$ is the order of diffraction, $\phi$ is the height of the phase step (a kind of the threshold level which should be equal to $\pi$ for the maximal possible diffraction efficiency), and $a$ is the fill factor. Considering different structures the general equation describing the amplitude coefficients of the expansion of the function describing the grating into a Fourier series should be used as follows:

$$
\begin{aligned}
& \eta_{0}=\left|G_{0}\right|^{2}=\left|\frac{1}{d} \int_{-d / 2}^{d / 2} f(x) d x\right|^{2}, \\
& \eta_{m}=\left|G_{m}\right|^{2}=\left|\frac{1}{d} \int_{-d / 2}^{d / 2} f(x) e^{-2 \pi i(m x / d)} d x\right|^{2} .
\end{aligned}
$$

Therefore, the exact theoretical calculation of diffraction efficiency is rather complicated and indirect for more advanced structures. However, the estimation may be carried out, because it is relatively easy to define it for simpler structure that would correspond to the less efficient case of complicated structure.

It should be underlined that fast changes of fill factor (like for narrow zones whose width is lower than the thickness of the structure) mostly introduce the decrease of efficiency in the range of only few percentages. Much more significant is the shadow effect [21] that can considerably decrease the efficiency especially for kinoform structures (for binary structures this effect is less important due to the smaller thickness of the structure).

Here, we concentrated our research on paper structures which are easy in fabrication, very cost-efficient, and fast in manufacturing. There are two methods which are worth mentioning. One of them is laser cutting [20] and it allows obtaining binary structures or eventually multistep phase structures (maximum 3-4 steps but it is wavelength dependent and corresponds to diffractive zones widths). The smallest feature is limited by the kerf of the laser cutting (which is the gap resulting from the finite laser spot size). The second method is $3 \mathrm{D}$ printing based on cutting the paper [25]. In this manufacturing method it is possible to perform automatic manufacturing of kinoforms, but its main drawback is the need of the thin substrate (around 1-2-mmthick) in order to get rid of local zero thickness and to guarantee proper stability of the structure [26]. A classical kinoform is a phase diffractive object which modulates the phase of incident light from the range $0-2 \pi$ in a continuous way. We can imagine that we take a refractive (thick) element and slice it into layers changing phase from 0 to maximally $2 \pi$ and then remove all parts (blocks) introducing exactly $2 \pi$ shift. In such way we will obtain a structure that is thin and has the same shape as refractive one but is formed with steps having some maximal height (corresponding to the $2 \pi$ phase shift), whereas a high order kinoform (HOK) is an optical structure that is made of zones similar to those in typical kinoform but having thickness corresponding to the phase shift of the multiplicity of $2 \pi$, and therefore having also different widths. In comparison to classical Fresnel lenses, such structure has better optical performance and is not suffering from chromatic aberration like first-order kinoform 
[5]. Therefore, even using diffractive optics we can work in broadband range.

3.1. Influence of Refractive Index on Designed Structure. Refractive index is very important for proper designing. In optics the refractive index of the material dependent on the frequency is mostly used; however one can also use defining structure parameters by its dielectric constant (if we assume small attenuation we can describe the refractive index as the square root of dielectric constant). For phase diffractive objects, the thickness of the structure corresponds to the phase change of the incident wave. The relation between the maximum thicknesses $h_{\max }$ of the structure for introducing phase shift by $2 \pi$ includes the influence of the used wavelength $\lambda$ and refractive indices of the surrounding medium $n_{0}$ and of the structure $n$ described with the following equation:

$$
h_{\max }=\frac{\lambda}{n-n_{0}} .
$$

That is why it is crucial to know the refractive index of the used material before designing the diffractive structure.

3.2. Absorption Coefficient. As it was mentioned, it is very important to know the optical parameters such as absorption coefficient and refractive index of the material (for designing diffractive optics we use values of $n$ and $\alpha$; however sometimes for $\mathrm{THz}$ and millimeter waves complex values are used) used to design the structure. Both these values can be precisely determined for many frequencies using Time Domain Spectroscopy [27]. Defining the refractive index is crucial to match the proper height (and design appropriate structure thickness) of the step of the designed structure, while absorption coefficient tells us about losses introduced by the structure. We can choose the material with smallest attenuation, but in the designing process we cannot suppress the influence of different absorption resulting from different thicknesses of the structure. We can simulate the result but similarly to the situation of nonuniform illumination we cannot change it.

The easiest way to determine the absorption coefficient for samples with known thickness $(h)$ is to measure the incident intensity of the light $I_{i}$ and transmitted intensity $I_{t}$. Then, the absorption coefficient can be calculated from the following:

$$
I_{t}(x)=I_{i} e^{-\alpha h} .
$$

It allows us to determine how large will be the influence of the attenuation on our designed structure and what differences of amplitude the structure will introduce. However, the case becomes more complicated when the sample is not flat or not homogeneous. According to this fact, it is difficult to establish its thickness in every point. In this case the simplest way to measure transmittance of the sample is by using the following:

$$
T=\frac{I_{t} \cos \left(\theta_{t}\right)}{I_{i} \cos \left(\theta_{i}\right)}
$$

TABLE 1: Values of refractive indices and absorption coefficients for frequency $0.3 \mathrm{THz}$. (g) corresponds to samples with glue.

\begin{tabular}{lcccc}
\hline Paper name & $h[\mathrm{~mm}] \pm 0.01 \mathrm{~mm}$ & $\alpha\left[\mathrm{cm}^{-1}\right]$ & $n$ & $\Delta n[\%]$ \\
\hline 3D_orientA & 3.97 & 2.190 & 1.399 & - \\
3D_orientB & 4.04 & 1.694 & 1.391 & - \\
3D_orientC & 4.14 & 1.752 & 1.395 & - \\
White & 0.63 & 2.122 & 1.488 & 0.33 \\
White (g) & 0.65 & 2.024 & 1.483 & \\
Glossy & 1.93 & 2.076 & 1.539 & 0.33 \\
Glossy (g) & 1.94 & 2.378 & 1.544 & \\
Black & 2.26 & 2.529 & 1.577 & 0.31 \\
Black (g) & 2.33 & 2.368 & 1.572 & \\
Gray & 1.75 & 2.153 & 1.546 & \\
Gray (g) & 1.77 & 1.852 & 1.552 & 0.37 \\
Crossed & 0.67 & 2.458 & 1.500 & 1.00 \\
Crossed (g) & 0.69 & 2.121 & 1.515 & \\
Green & 2.62 & 2.037 & 1.436 & \\
Green (g) & 2.62 & 2.029 & 1.434 & 0.14 \\
Green (g) + food foil & 2.69 & 2.008 & 1.427 & 0.58 \\
Green (g) + office foil & 2.71 & 2.071 & 1.431 & 0.31 \\
Green (g) + thick foil & 2.70 & 2.058 & 1.437 & 0.11 \\
\hline
\end{tabular}

where $\theta_{i}$ and $\theta_{t}$ are the angle of incidence and the angle of refraction, respectively. Both are measured according to the normal to the surface. Then we can make transmission maps and verify what is the influence of variable attenuation on the designed structure.

Nevertheless, the thickness of the structure should be relatively small to enable efficient performance and the proper material should be used.

In reality we must always assume that we are dealing with amplitude and phase structures, whose transmittance is described with the following equation:

$$
T=A(x, y) e^{i \varphi(x, y)} .
$$

So even if we are designing only phase structure, we must deal with its amplitude contribution (due to the absorption of the used material).

3.3. Analyzed Papers. Because there are a lot of different kinds of papers available on the market, it is possible to choose the best one in terms of properties such as absorption coefficient and refractive index, but also stiffness and other crucial parameters. We have examined some samples that are listed in Table 1 and their values of refractive indices and absorption coefficients for the frequency corresponding to the design wavelength $(0.3 \mathrm{THz})$ are determined. Table 1 contains the name of each paper, the thickness of the sample (measured with the digital micrometer screw with accuracy $0.001 \mathrm{~mm}$, but taking into account flexibility of the material we assumed that the accuracy was $0.01 \mathrm{~mm}$ ), its absorption coefficient, and refractive index. The last column includes the difference between the refractive indices of the paper with and without glue (given in percent) that shows that using glue does only slightly change paper parameters. 
The efficient numerical simulation which is consistent with experimental results requires initial determination of parameters of used material, like refractive index and absorption coefficient. Therefore, different paper samples were investigated by means of Teraview TPS Spectra 3000 spectrometer. THz optical setup was used in the transmission mode and allowed determining paper material parameters for the available part of $\mathrm{THz}$ frequency range. Measurements were performed in humidity controlled environment (dried air with the humidity not exceeding $0.5 \%$ ). The aging of paper does not influence the measurements during few years period. The described green paper was verified in 2011 for the first time [20] and now the new green paper and the old one have same optical properties.

First group of papers are (3D_orientA, 3D_orientB, and 3D_orientC) manufactured by 3D printing technique (Mcor IRIS printer [25]) from Xerox white paper with paper substance (grammage) of $80\left(\mathrm{~g} / \mathrm{m}^{2}\right)$. Due to the manufacturing process they are already glued but samples have different orientation of paper layers, which changes their attenuation coefficient. The refractive index values are relatively the same (change smaller than $0.6 \%$ ). The orientation described here is the sequence of gluing and cutting paper sheets in $3 \mathrm{D}$ printing technique, which should not introduce birefringence.

The rest of the samples were analyzed in two different configurations: layers of paper, stacked on each other without and with adhesive bonding (3M Display mount glue). In Table 1 we described optical parameters of different papers with abbreviated names and here we will give more details about them. Papers 3D_orient were already described (Xerox white paper $\left.80\left(\mathrm{~g} / \mathrm{m}^{2}\right)\right)$. White paper is commercially available Lyreco white paper with grammage also $80\left(\mathrm{~g} / \mathrm{m}^{2}\right)$. Glossy is Sirio Pearl paper manufactured by Fedrigoni in Ice White color with grammage $300\left(\mathrm{~g} / \mathrm{m}^{2}\right)$. Black is Burano paper manufactured by Favini in Nero color with grammage 320 $\left(\mathrm{g} / \mathrm{m}^{2}\right)$. Gray is cardboard folder manufactured by Hamelin Top 2000 in White color with grammage $350\left(\mathrm{~g} / \mathrm{m}^{2}\right)$. Crossed is notebook squared paper manufactured by Basic in White color. Green is Curious Metallics paper from Arjo Wiggins in Jaspis color and $300\left(\mathrm{~g} / \mathrm{m}^{2}\right)$. Furthermore, for green paper (which was used to create diffractive lens [28]), additional measurements were carried out for different types of foils, that could be used to create hermetic seals on the paper (nullifying the effect of humidity). As we can see the most similar values of both compared parameters have green paper; measurements for all TDS frequency range are shown in Figure 1.

For green paper both refractive index and absorption coefficient curves are smooth (Figure 2). When we assume that paper is not resistant to water we can imagine putting such structures into foil and sealing it inside. Therefore, we examined the influence of adding the foil on values of refractive index and absorption coefficient. All values differed less than $0.6 \%$ and both the shape of the curve and its tendency did not change. This difference of refractive indices is mostly the result of changing the thickness of the analyzed structures (foil is almost transparent for $\mathrm{THz}$ radiation, so paper sample with added foil layers has bigger thickness but its attenuation remains more or less the same). In case

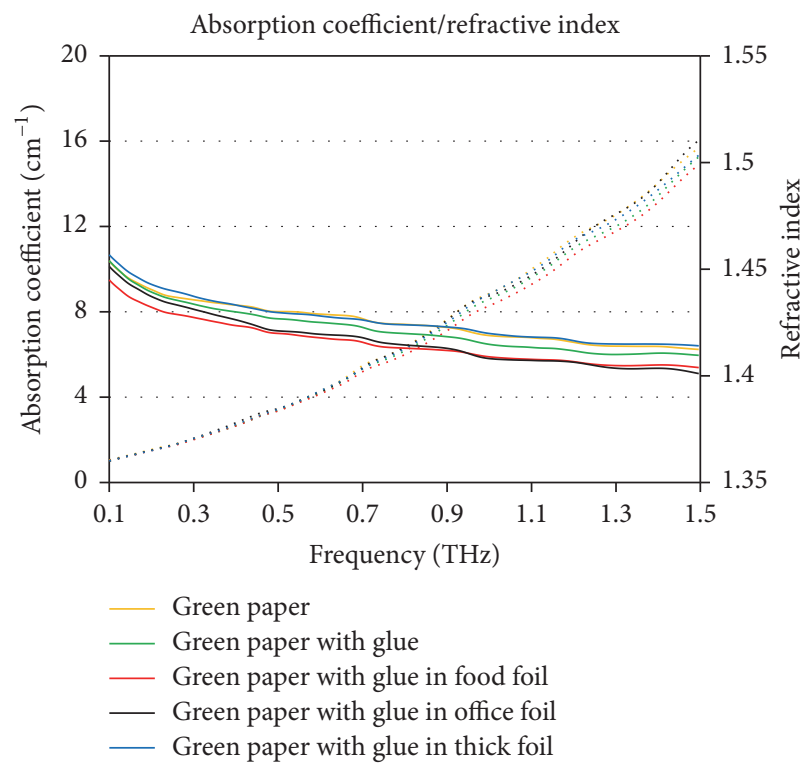

FIGURE 1: Absorption coefficient (dotted lines) and refractive index (full lines) curves for green paper with different additions.

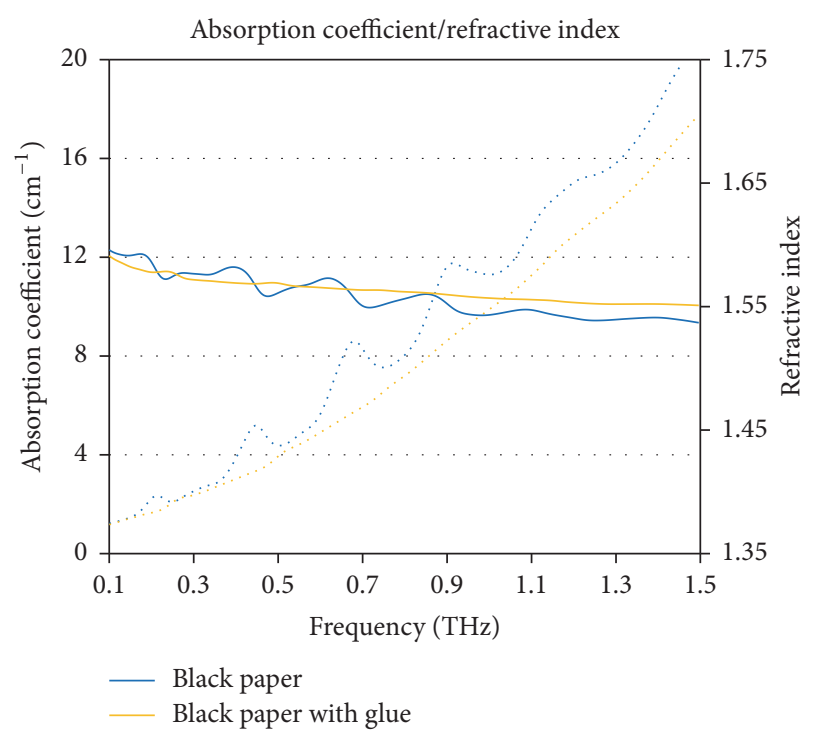

FIGURE 2: Absorption coefficient (dotted lines) and refractive index (full lines) curves for black paper with and without glue.

TABLE 2: The variation of the refractive index and the absorption coefficient for $0.3 \mathrm{THz}$ for green paper with determined inaccuracy.

\begin{tabular}{lc}
\hline Refractive index $n$ & Absorption coefficient $\alpha\left[\mathrm{cm}^{-1}\right]$ \\
\hline $1.432 \pm 0.050(3.5 \%)$ & $2.040 \pm 0.032(1.6 \%)$ \\
\hline
\end{tabular}

of absorption coefficient, there is no visible difference for design wavelength of $1 \mathrm{~mm}$ (corresponding to $0.3 \mathrm{THz}$ ). The variation of refractive index and absorption coefficient are gathered and we determined one value with its inaccuracy for both parameters, which are shown in Table 2. 


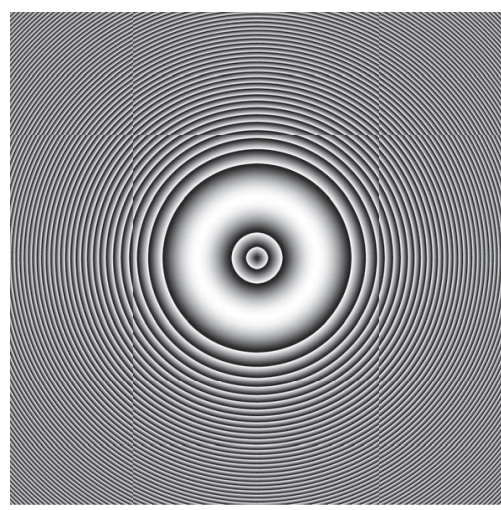

(a)

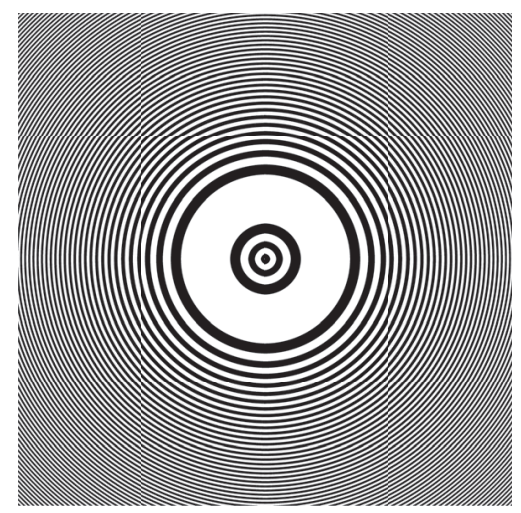

(b)

Figure 3: The phase distribution of the ideal diffractive kinoform (a) and binary (b) lens structure designed for the plane wave illumination, where white areas introduce $2 \pi$ (a) and $\pi$ (b) phase shift and black do not change the phase.

For higher frequencies $(>0.7 \mathrm{THz})$ absorption coefficients for different samples of green paper with foil start varying from each other. However, it is important to remember that the higher the frequency is (around $1.5 \mathrm{THz}$ and higher), the more noisy the absorption curve becomes; thus the best measurements are conducted for lower frequencies. Although there is a possibility to measure absorption coefficient and refractive index for frequencies higher than $0.7 \mathrm{THz}$, we must realize that for this range these are only amplitude structures (due to the high attenuation). For higher frequencies it is advisable to use other materials as phase structures, while for lower frequencies the paper seems to be good material. Therefore, due to the growing attenuation of paper for higher frequencies we decided to use $0.3 \mathrm{THz}$ which corresponds to the DWL $=1 \mathrm{~mm}$. Such radiation can be used in $\mathrm{THz}$ scanning as we had already demonstrated but finally using structure from polyamide [29].

In case of paper structures consisting of more than one layer (to assure proper height of the step) we must ensure proper manufacturing in case of geometry and gluing to avoid unwanted effects resulting from spurious air layers between paper layers. Such phenomenon may be observed for black paper shown in Figure 2.

It can be seen that for black paper there is significant difference between two investigated configurations (with and without glue). This effect is present in both refractive index and absorption coefficient curves (Figure 3) in the form of following peaks appearing for multiplicities of first frequency (around $0.2 \mathrm{THz}$ ). Frequencies for which characteristic peaks appear are as follows: $0.21 ; 0.44 ; 0.68$; and $0.91 \mathrm{THz}$. It can be easily noticed, that structure without adhesive bonding acts similarly to Fabry-Perot resonator which is reflecting resonant frequencies, whereas for glued structure this effect disappears. We do not know if the attenuated frequencies are reflected back from the paper sample or attenuated and for that reason some additional measurements should be performed but since we want to avoid this effect we will not continue these considerations.

\section{Exemplary Structure}

To demonstrate the applicability of the paper for designing diffractive structures, a toroidal lens was made from one of the tested papers [26]. The simulations were carried out for the optical setup used in the experiment and shown in Figure 11 .

The toroidal lens instead of focusing light in one focal point like normal lens forms a ring with predefined diameter in the focal plane. In an ideal case the diffractive structure was designed in the form of kinoform having continuous phase changes distribution and no attenuation which is shown in Figure 3.

For the simplicity we manufactured the structure by laser cutting, therefore we used the binary phase element which contains the correcting structure for the divergent wavefront coming from the pinhole. The phase distribution of the designed element assuming the illumination from a pinhole is shown in Figure 4.

Such structure should redirect the incident light in the light ring in the focal plane. Unfortunately, in real case, it is almost impossible to create perfect kinoforms which would introduce only well-adjusted phase change without any attenuation. Hence in order to facilitate manufacturing the designed structure was recalculated into binary structure using paper as a material (obtaining good results means the possibility to manufacture paper DOE using laser cutting or $3 \mathrm{D}$ printing techniques). Another problem is that it is impossible to create structures "hanging in air." According to this inconvenience, all manufactured binary structures possess additional supporting bars or additional substrate layer. Those reinforcements introduce some distortions in obtained energy distributions, which can be seen as bright spots around the ring, but they introduce less attenuation in comparison with additional layer of material.

The manufactured structure was scanned in order to carry out simulations for toroidal diffractive lens with additional reinforcements (Figure 5). 


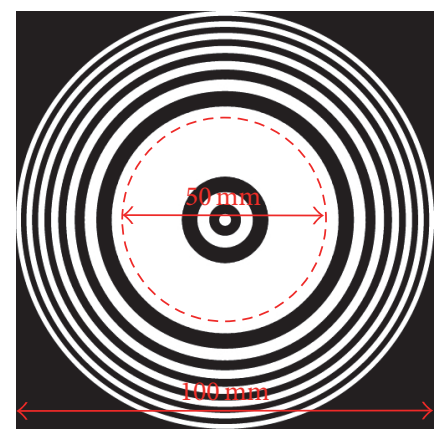

Figure 4: The phase distribution of the ideal diffractive lens structure, where white areas introduce $\pi$ phase shift and black do not change the phase.

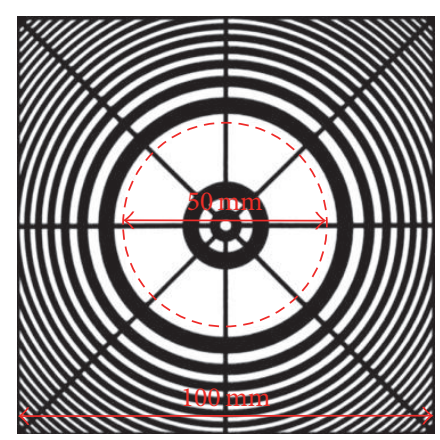

FIGURE 5: The phase distribution of the designed and manufactured diffractive lens structure (scanned), where white areas introduce $\pi$ phase shift and black do not change the phase.

\section{Simulations}

Simulations were performed by means of modified convolution method $[30,31]$ on $4096 p x \times 4096 p x$ matrices with sampling $50 \times 50 \mu \mathrm{m}^{2}$. The tested structure was designed for wavelength $\lambda=1 \mathrm{~mm}$, which corresponds to the frequency $0.3 \mathrm{THz}$. The designed lens had focal length $f=100 \mathrm{~mm}$ and was illuminated with divergent wave coming from the pinhole $(2.5 \mathrm{~mm}$ diameter). The pinhole was placed $300 \mathrm{~mm}$ before the structure and the resulting wave illuminated most of the structure almost uniformly.

Here, we want to carry out simulations showing the influence of manufacturing problems on obtained results and the possibility of simulating different real conditions mostly present in experimental setups.

Two types of input data were prepared for simulations: ideal and scanned diffractive element, shown in Figures 4 and 5. First structure is an ideal representation of designed diffractive lens-like structure, whereas the second one is the scanned image of real, manufactured object. The difference between those two structures lies in the existence of suspending bars, which are required to keep different phase changing areas together.

Three simulations were done for these two objects: one considering the possibility of manufacturing not-exact phase step height (Figures 6 and 7), second assuming the attenuation of the elements made from paper (Figures 8 and 9), and third taking into account that the beam illuminating the structure has Gaussian-like intensity shape with different diameters (which corresponds to the nonuniform illumination, Figure 10).

In the first series of simulations the influence of phase change mismatch was checked. This was done by changing the phase delay introduced by the phase object. In real case introduced phase delay corresponds to the thickness of the object; thus this simulation allows investigating the effect of manufacturing process precision or rather its imprecision (regarding not matched thickness) on the obtained efficiency. Here, we assume uniform amplitude over the whole structure and we analyze only the varying value of the phase step value $\phi$ corresponding to the height of the phase step introducing $\pi$ phase shift (Figure 6). The intensity distributions of simulated structures are shown in Figures 6(a)-6(i).

Next, we assume the same uniform amplitude over the whole structure with varying phase step value $\phi$ for the manufactured structured. After preparing the design of the structure with supporting bars, it was manufactured by laser cutting and then scanned to perform the simulations verifying its correctness. The results obtained in Figure 7 show intensity distributions for the scanned structure for different phase delays that correspond to the one calculated for the ideal structure (Figure 6).

Results show that due to the phase mismatch, the uniform intensity distribution inside the circle changes and we can observe either bright or dark dots in the middle (depending on the phase mismatch) that do not appear for the ideal toroidal lens. This effect is almost not observable in simulation results for scanned object; thus it is possible to say that small phase delay mismatching is not a crucial factor in proper functioning of the diffractive lens. Simulation results for scanned object also reveal the fact that more energy is focused in particular points on the ring. Those "defects" are caused by existence of suspending bars.

The second simulation was conducted in order to investigate how attenuation/absorption of paper affected output focal curve of the diffractive lens. This was done by changing the attenuation of the structure in regions where phase was changed, under the assumption of structure uniformity (Figure 8). These regions had lower amplitude in comparison with regions only with air (having $A=1$ ).

These intensity distributions do not vary in shape and the difference is hard to notice (Figure 8). In case of scanned structure simulation, the obtained intensity distributions are also very similar (Figure 9).

Due to the fact that there are no real visible differences between simulation results for different attenuations of the structure material, we performed the quantitative comparison. As it can be seen the attenuation does not significantly affect the way lens works. However, it introduces changes in the amount of focused light, which can be seen from integral values presented in Table 3. For each intensity distribution we have calculate the total intensity of all pixels forming focal curve and then normalized it to the value of ideal structure without assumed attenuation (like in Figure 8(c)), which was also the maximal value for all cases. 

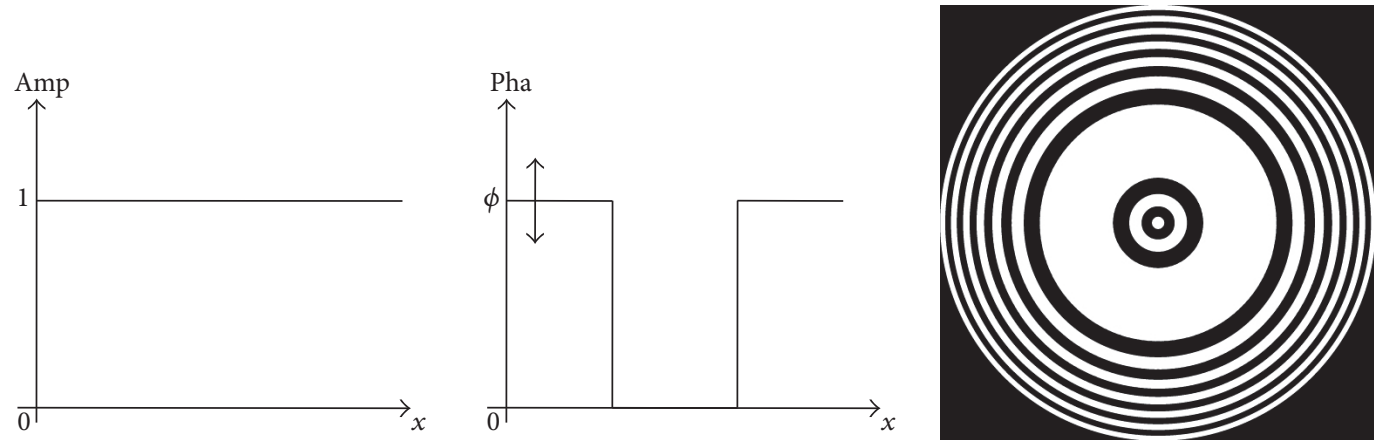

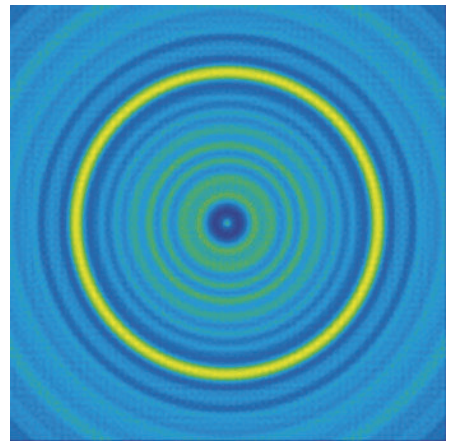

(a) $\phi=0.3$

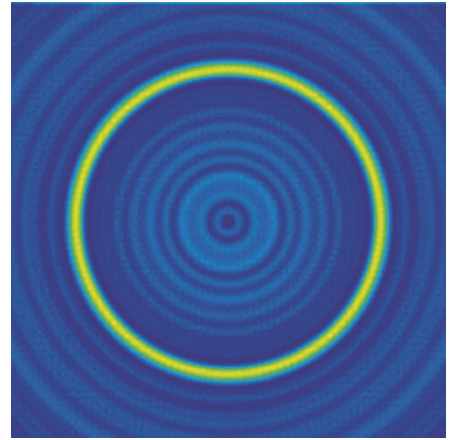

(d) $\phi=0.9$

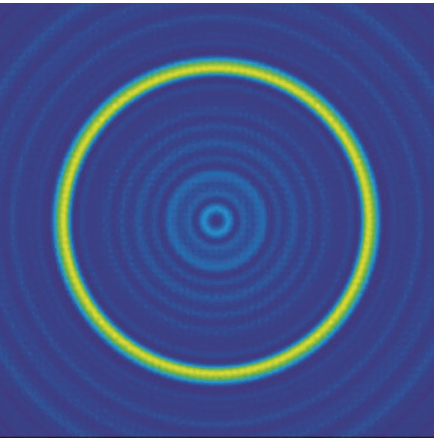

(g) $\phi=1.3$

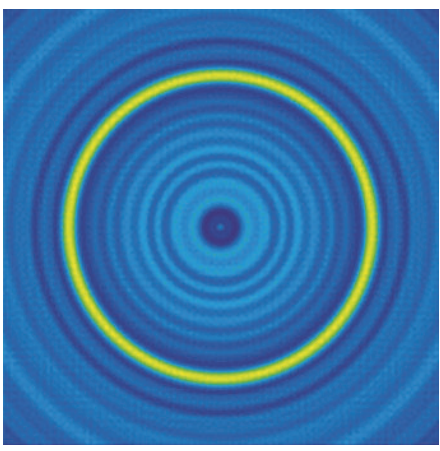

(b) $\phi=0.5$

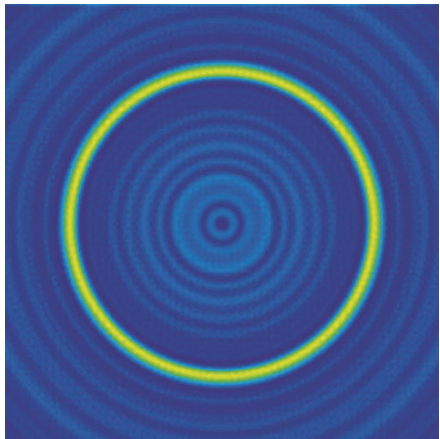

(e) $\phi=1.0$

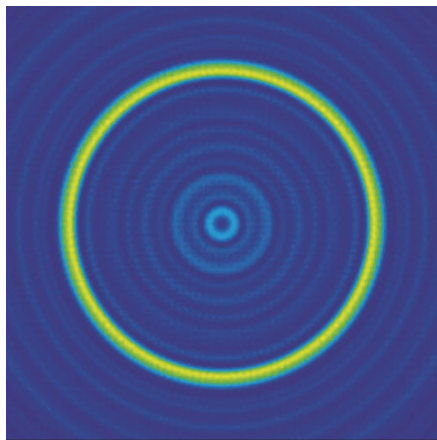

(h) $\phi=1.5$

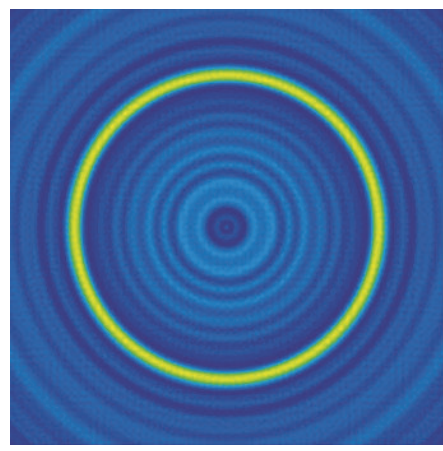

(c) $\phi=0.7$

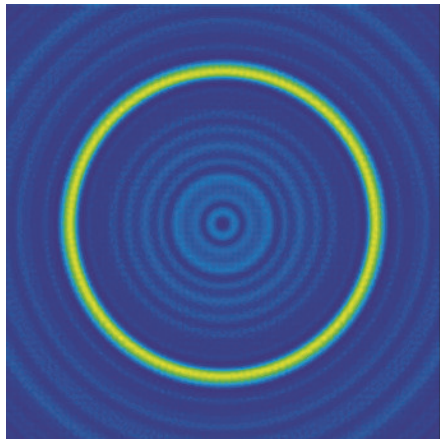

(f) $\phi=1.1$

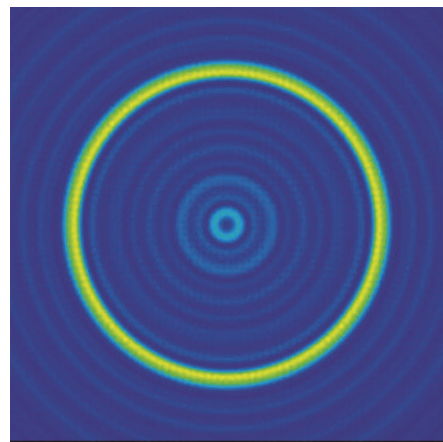

(i) $\phi=1.6$

Figure 6: The simulation parameters (amplitude and phase diagrams together with the structure's phase distribution). Intensity distributions (simulation results) for different phase mismatch for ideal diffractive lens. Simulations were conducted for introduced phase delays as follows: $0.3,0.5,0.7,0.9,1.0,1.1,1.3,1.5$, and 1.6 , shown in (a)-(i), respectively. 

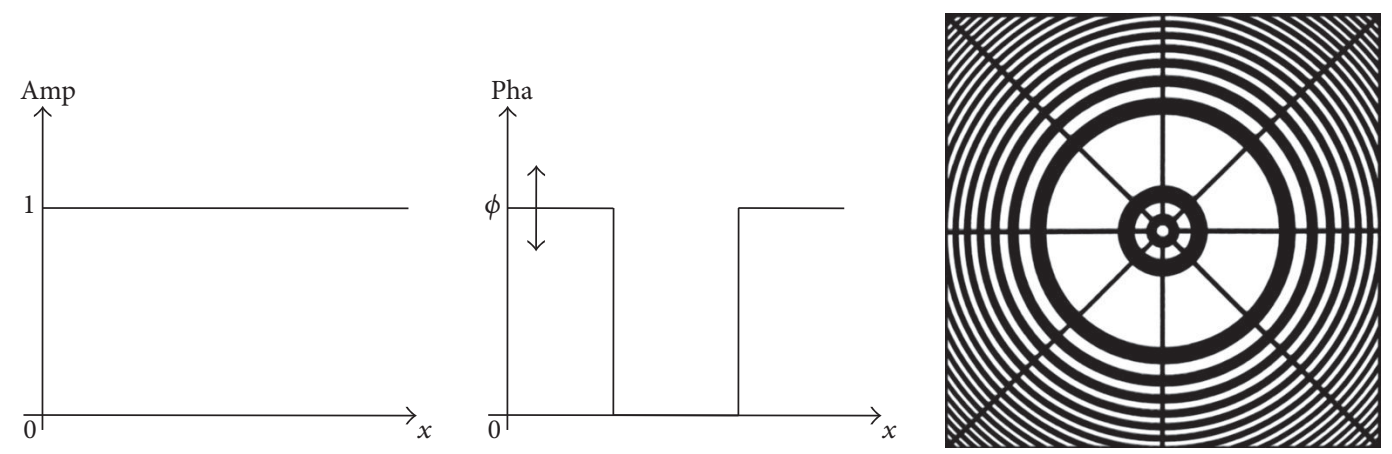

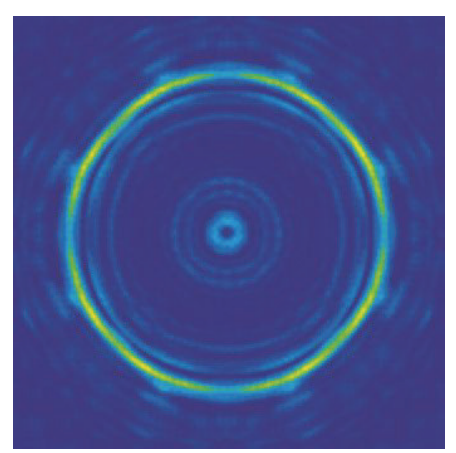

(a) $\phi=0.8$

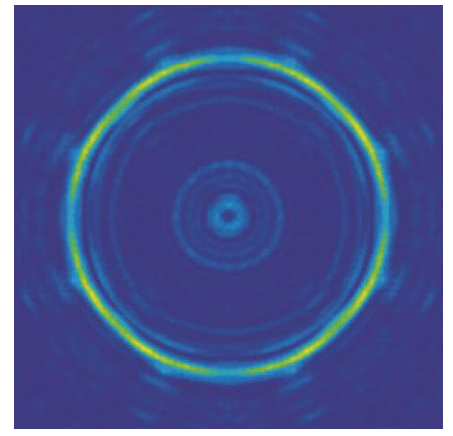

(d) $\phi=0.95$

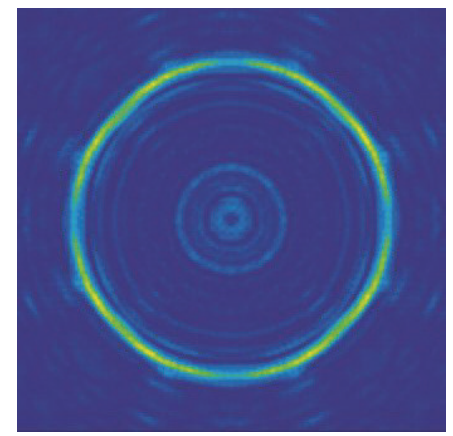

(g) $\phi=1.1$

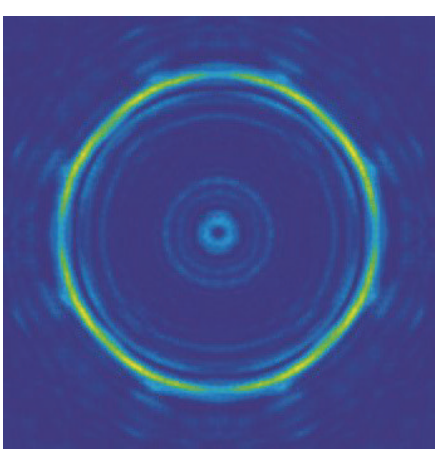

(b) $\phi=0.85$

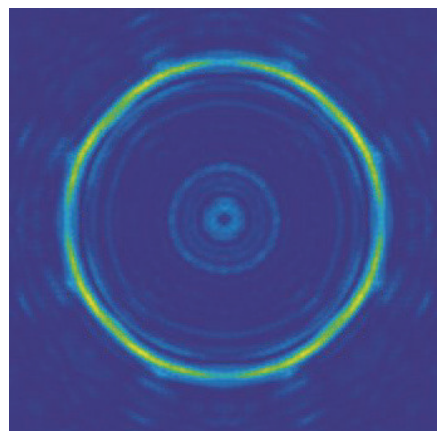

(e) $\phi=1.0$

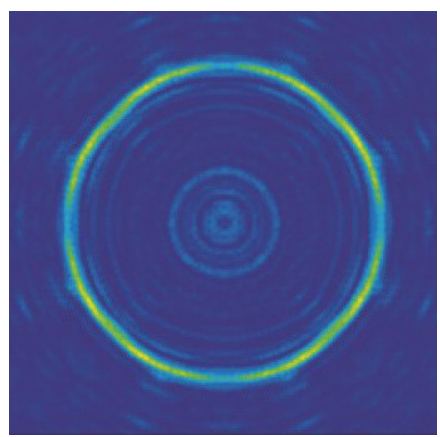

(h) $\phi=1.15$

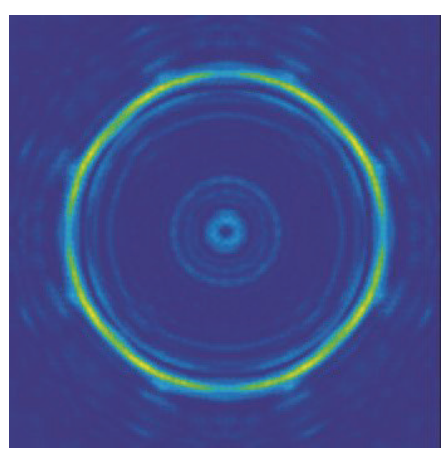

(c) $\phi=0.9$

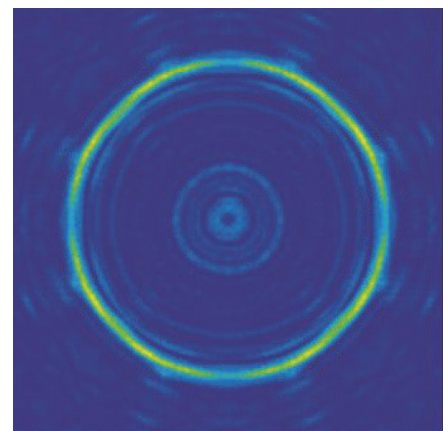

(f) $\phi=1.05$

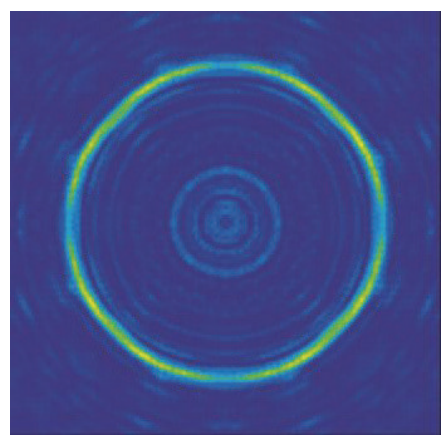

(i) $\phi=1.2$

FIGURE 7: The simulation parameters (amplitude and phase diagrams together with the structure's phase distribution). Intensity distributions (simulation results) for different phase mismatch for scanned structure. Simulations were conducted for introduced phase delay as follows: $0.8,0.85,0.9,0.95,1.0,1.05,1.1,1.15$, and 1.2 , shown in (a)-(i), respectively. 

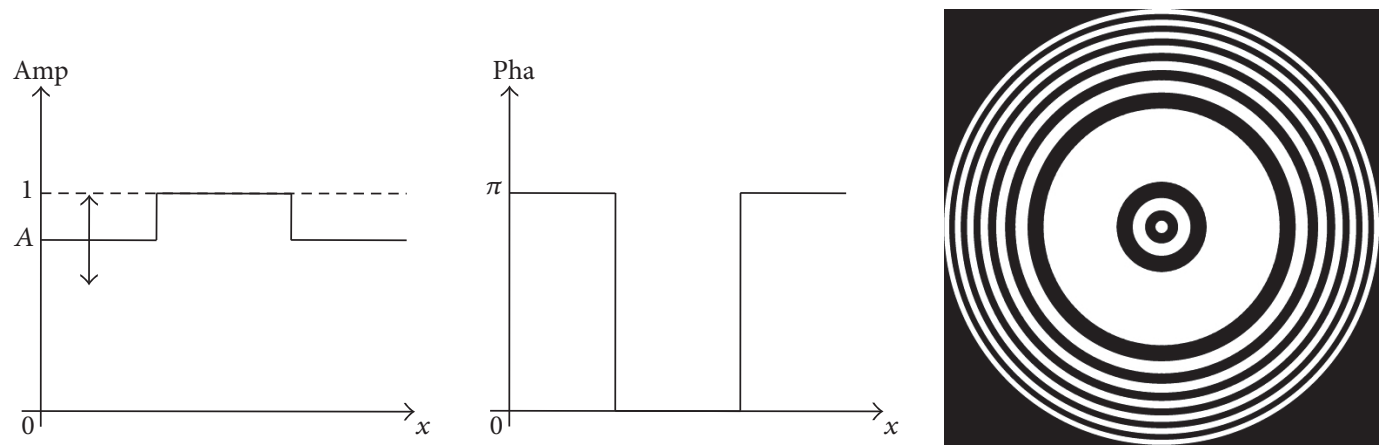

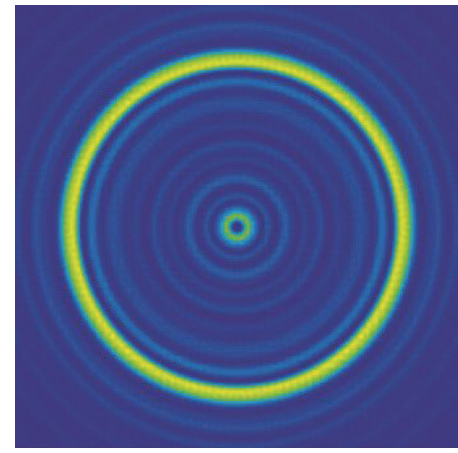

(a) $A=0.8$

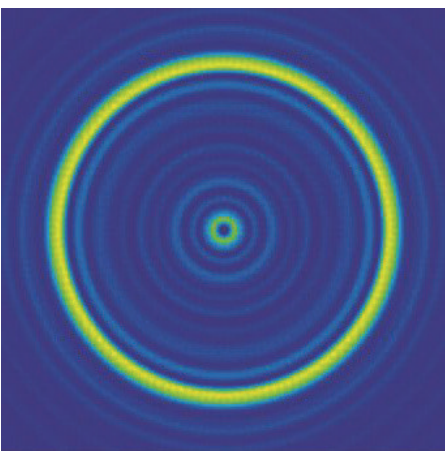

(b) $A=0.9$

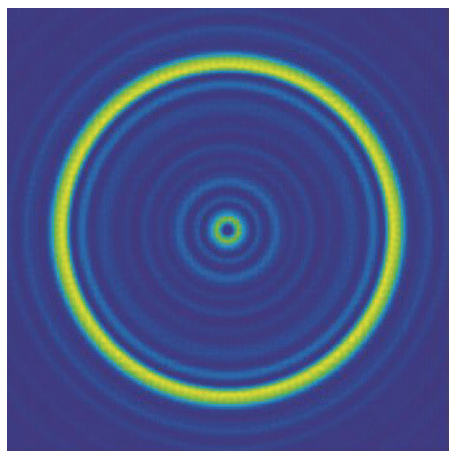

(c) $A=1.0$

FIGURE 8: The simulation parameters (amplitude and phase diagrams together with the structure's phase distribution). Intensity distributions (simulation results) for different material attenuation for ideal lens. Simulations were conducted for material attenuation values (1.0 value corresponds to no attenuation situation) as follows: $0.8,0.9$, and 1.0 , shown in (a)-(c), respectively.
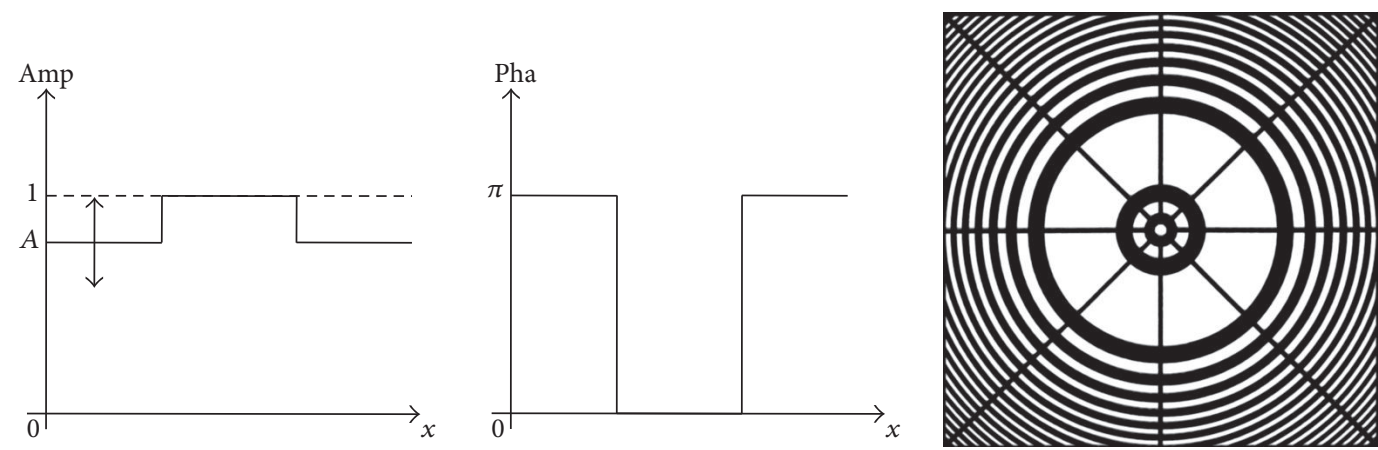

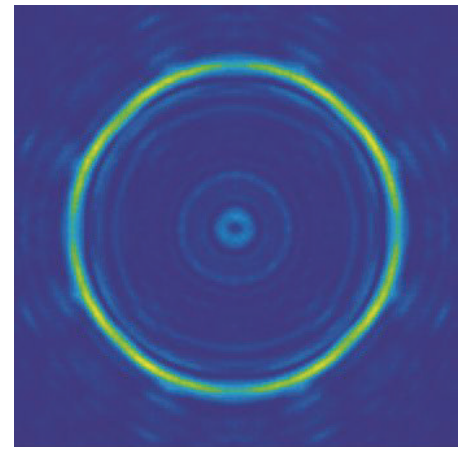

(a) $A=0.8$

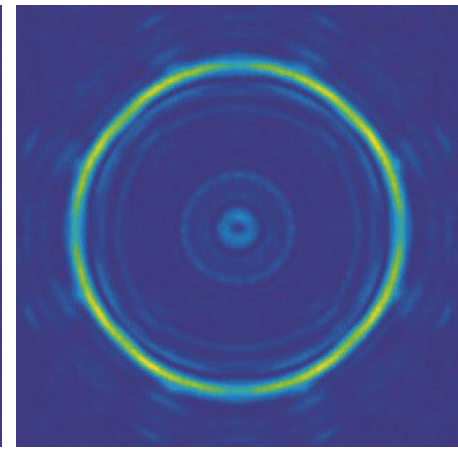

(b) $A=0.9$

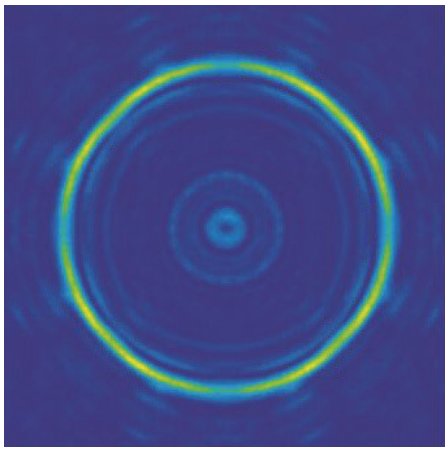

(c) $A=1.0$

FIGURE 9: The simulation parameters (amplitude and phase diagrams together with the structure's phase distribution). Intensity distributions (simulation results) for different material attenuation for scanned lens. Simulations were conducted for material attenuation values (1.0 value corresponds to no attenuation situation) as follows: $0.8,0.9$, and 1.0 , shown in (a)-(c), respectively. 

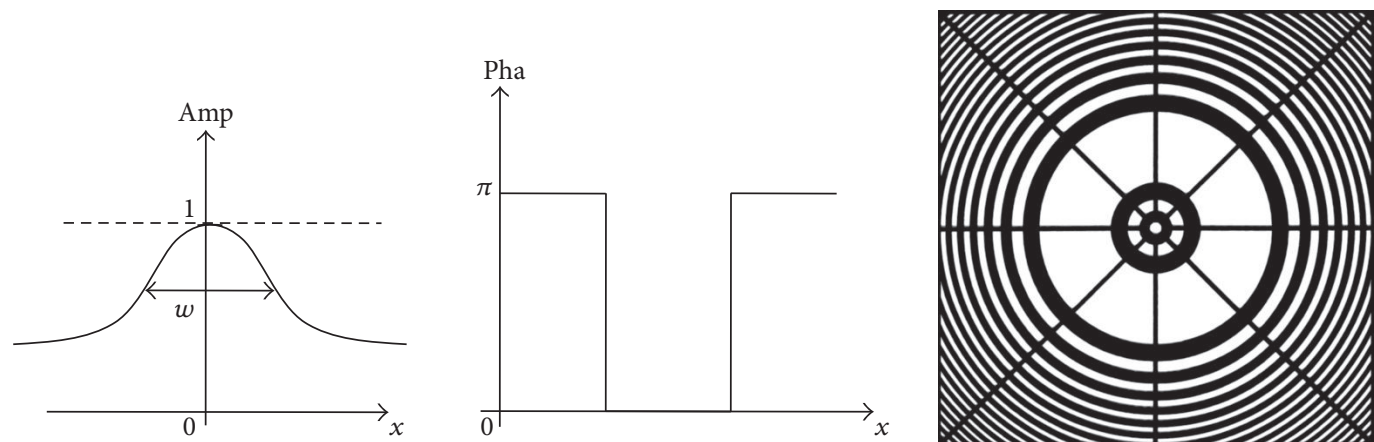

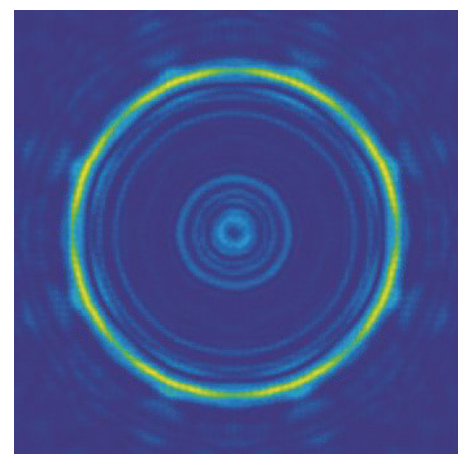

(a) Plane wave

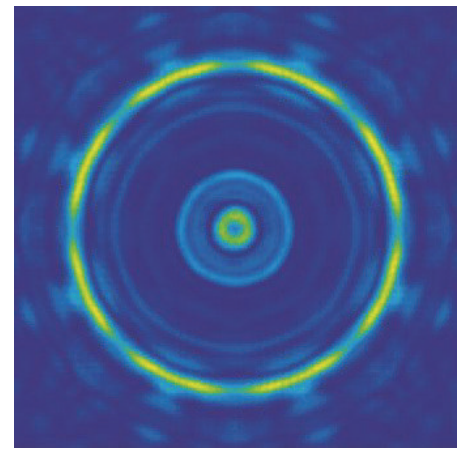

(d) $w=40 \mathrm{~mm}$

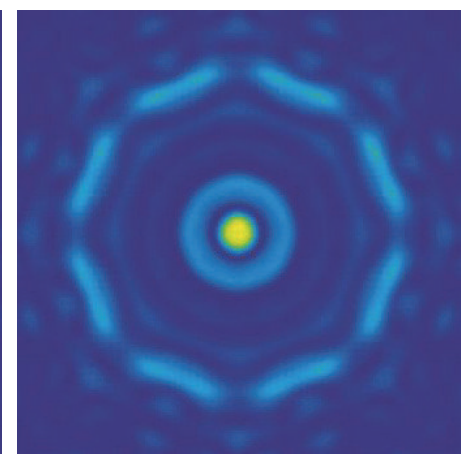

(b) $w=20 \mathrm{~mm}$

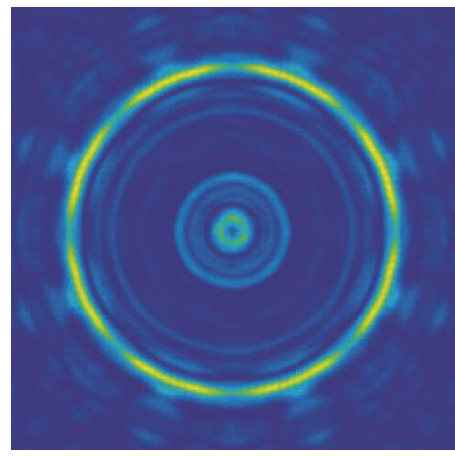

(e) $w=50 \mathrm{~mm}$

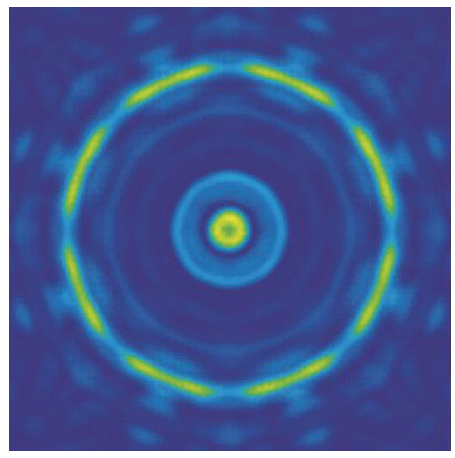

(c) $w=30 \mathrm{~mm}$

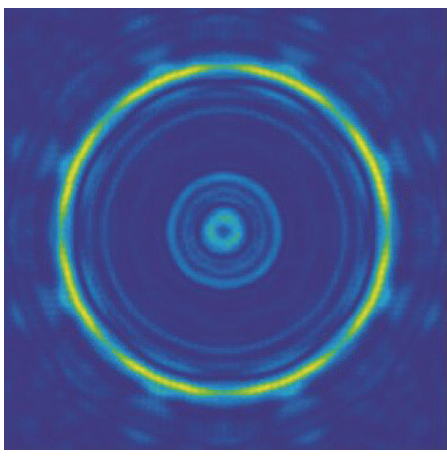

(f) $w=60 \mathrm{~mm}$

FIGURE 10: The simulation parameters (amplitude and phase diagrams together with the structure's phase distribution). Intensity distributions (simulation results) for different lens illumination. Diffractive structures were illuminated with Gaussian beams with different waist values $w$ (in $\mathrm{mm}$ ) as follows: 20,30,40,50, and 60, shown in (b)-(f), respectively. In case of sufficient illumination (plane wave) (a).

Now, the difference is clearly seen. The ideal structure has $15 \%$ less energy in the focal plane when we assumed the amplitude in the paper region equal to 0.8 and not to 1 . For scanned structure we can see significant decrease of the total intensity in the focal plane in comparison with ideal lens. It is caused mainly by the intensity disturbances introduced by supporting bars. They were designed as radial lines which can be without problems replaced with random distribution of bars. It would probably lower the overall intensity, but there would not be "defects" visible now in the form of bright dots in the ring.

In most cases when we design diffractive structure we assume uniform illumination (plane wave). Unfortunately, in reality it is not possible to obtain perfectly uniform illumi-
TABLE 3: Collected light according to value of the integral over the whole image.

\begin{tabular}{lccc}
\hline Total intensity [a.u.] & $A=0.8(\mathrm{a})$ & $A=0.9(\mathrm{~b})$ & $A=1(\mathrm{c})$ \\
\hline Ideal structure & 0.85 & 0.93 & 1.00 \\
Scanned structure & 0.55 & 0.60 & 0.65 \\
\hline
\end{tabular}

nation. We cannot simply change the shape of the beam but we can simulate the influence of those irregularities.

Third comparison was carried out in order to analyze the effect of illumination of the designed lens with beam size different from lens diameter. This was done for illumination with Gaussian-like beam having different waist values $w$. 


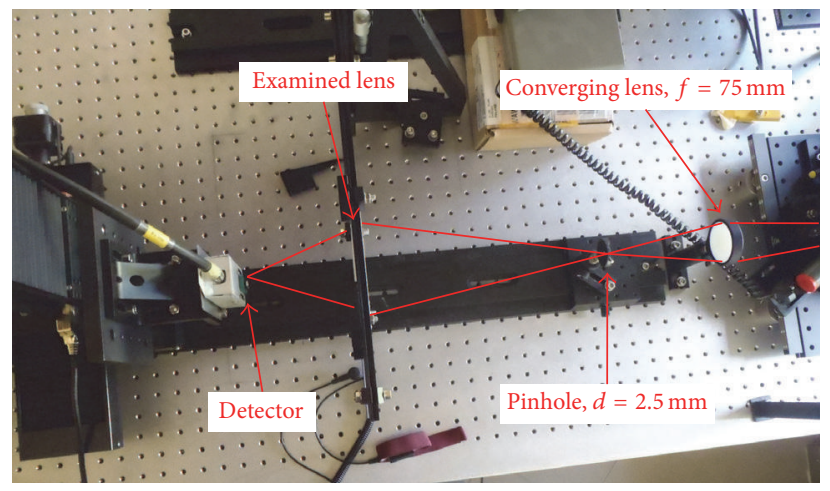

(a)

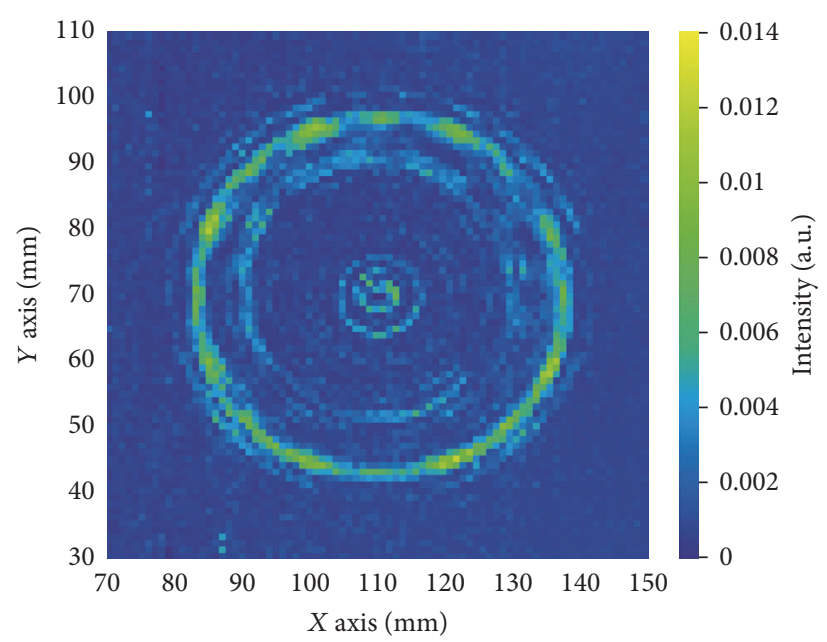

(b)

FIGURE 11: The experimental setup (a) and the intensity distribution registered in the focal plane (b).

Results clearly show that illumination of diffractive lens has a crucial impact on the functionality of the structure. Lens illuminated with smaller beam does not work as designed. It is caused by the fact that only part of the functioning area is illuminated; thus only part of it is working. For insufficient diameter of the illuminating beam (like in Figure 10(b)) we even cannot see the focusing into a circle. With the increase of illuminated area, the diffractive structure starts to operate as it was designed. For Gaussian beam with waist value bigger than the diameter of the lens, there is no visible change, as the structure is fully illuminated. Overall illumination is a crucial factor for diffractive elements to work properly, and moreover it may be inferred that the proper illumination of the structure is more crucial than the exact values of the structure thickness considering variable attenuation.

\section{Manufactured Structure-Results}

According to the results obtained from simulations the experimental evaluation was carried out. To test the structure, simple setup was used (Figure 11) based on the Schottky diode as a source and semiconductor transistor mounted on $X Y Z$ stage as the movable detector. In order to ensure as uniform as possible illumination of the structure, the $\mathrm{THz}$ wavefront from the source was modified with converging lens $(f=$ $75 \mathrm{~mm})$ and a pinhole $(d=2.5 \mathrm{~mm})$. The divergence of the beam was corrected by the designed structure and it focused the radiation $100 \mathrm{~mm}$ after the structure (in the focal plane). The experimental setup is illustrated in Figure 11(a) together with the registered amplitude distribution recalculated the intensity.

The experimentally obtained result (Figure 11) prove that designed and manufactured toroidal diffractive lens-like structure can be used in order to modify the $\mathrm{THz}$ wavefront. To show the good accordance between the theoretical simulation and experimental evaluation we have shown the experimental distribution, with simulation of scanned structure and ideal structure in Figure 12.

Simulations conducted for scanned structure (Figure 12(b)) are giving almost the same shape as obtained in the experiment (Figure 12(a)). What is worth mentioning is the fact that real focal plane was created at designed distance. This conformity is important for further attempts in designing new optical structures for particular applications. 


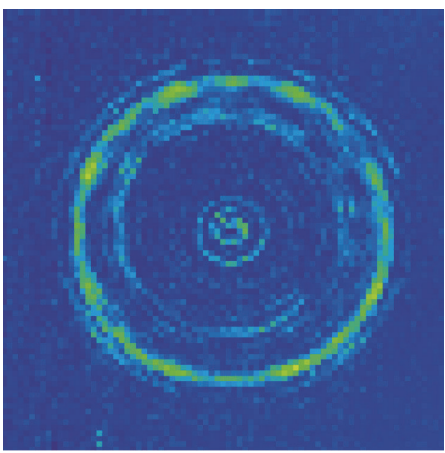

(a)

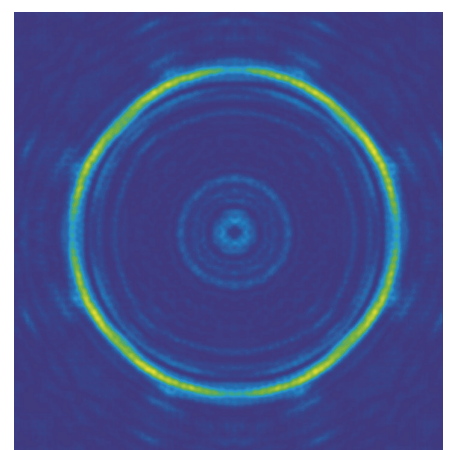

(b)

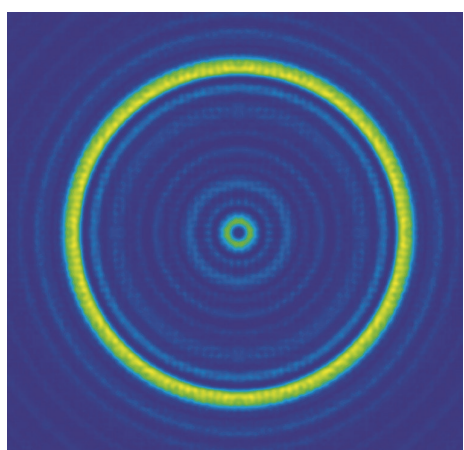

(c)

FIGURE 12: Experimental results (a), compared to simulation results of scanned structure (b) and ideal case (c).

The experimental evaluation shows that with the knowledge about material which was used for manufacturing the structure, it is possible to perform simulations before actually producing desired object. Thanks to that it is possible to avoid production of many structures with bad parameters and that opens new possibilities for bigger control over the THz beam.

\section{Conclusions}

Paper occurs to be very good material for $\mathrm{THz}$ radiation. Manufacturing paper optical elements is cheap and fast; there are two simple methods which allow producing good quality diffractive structures. Simulations carried out by means of modified convolution method allow properly foreseeing the behavior of the radiation. It was possible to obtain great accordance between numerical simulations and experimental evaluation. However, it is crucial to know the parameters of the used material before performing the simulations. It was proven that using adhesive bonding allows creating structures from multiple layers of paper without resonating effects. The addition of glue not always improves the efficiency but for the case of arising resonant frequencies can significantly help to suppress this unwanted effect.

Moreover, we suggest using suspension bars in random places to suppress their influence on the intensity distribution in the focal plane of the designed element.

Using paper as material for designing and manufacturing diffractive elements allows fast prototyping and according to obtained results such structures can successfully modify the $\mathrm{THz}$ wavefront. What is more, paper structures are cheap and can be used also to manufacture different optical elements [32].

Here, we described manufacturing of optical element for $\mathrm{THz}$ radiation from paper and the example of toroidal binary structure focusing in a focal circle. However, optical structures can be manufactured from paper with continuous phase profile (like kinoform structures) by 3D printer [25] which was demonstrated and compared in [26], even though the 3D printed lens must have substrate layer the kinoform structure has bigger efficiency than the binary lens manufactured from green paper described here.

\section{Competing Interests}

The authors declare that they have no competing interests.

\section{Acknowledgments}

This work was partially supported by National Center of Research and Development (NCBR) Grant LIDER/020/319/ L-5/13/NCBR/2014. The authors would like to thank Dr. Norbert Palka from Institute of Optoelectronics of Military University of Technology for the possibility of performing the measurements in Laboratory of Terahertz Technique. The authors would also like to thank the Orteh Company for providing LS 6.0 Software used for designing and modeling diffractive optical elements.

\section{References}

[1] N. Palka, "Identification of concealed materials, including explosives, by terahertz reflection spectroscopy," Optical Engineering, vol. 53, no. 3, Article ID 031202, 2014.

[2] N. Palka and D. Miedzinska, "Detailed non-destructive evaluation of UHMWPE composites in the terahertz range," Optical and Quantum Electronics, vol. 46, no. 4, pp. 515-525, 2014.

[3] V. A. Trofimov and S. A. Varentsova, "About efficiency of identification of materials using spectrum dynamics of medium response under the action of $\mathrm{THz}$ radiation," in Terahertz Physics, Devices, and Systems III: Advanced Applications in Industry and Defense, vol. 7311 of Proceedings of SPIE, p. 73110U, Orlando, Fla, USA, April 2009.

[4] J.-H. Son, Terahertz Biomedical Science \& Tehcnology, CRC Press, Boca Raton, Fla, USA, 2014.

[5] J. Suszek, A. M. Siemion, N. Blocki et al., "High order kinoforms as a broadband achromatic diffractive optics for terahertz beams," Optics Express, vol. 22, no. 3, pp. 3137-3144, 2014.

[6] http://www.tydexoptics.com/pdf/THz_Materials.pdf.

[7] http://www.zeonex.com/optics.aspx.

[8] S. Busch, M. Weidenbach, J. C. Balzer, and M. Koch, "THz optics 3D printed with TOPAS," Journal of Infrared, Millimeter, and Terahertz Waves, vol. 37, no. 4, pp. 303-307, 2016.

[9] J. R. Middendorf, D. A. LeMaster, M. Zarepoor, and E. R. Brown, "Design of multi-order diffractive THz lenses," in Proceedings 
of the 37th International Conference on Infrared, Millimeter, and Terahertz Waves (IRMMW-THz '12), pp. 23-28, THz Waves, Wollongong, Australia, September 2012.

[10] M. Sypek, M. Makowski, E. Hérault et al., "Highly efficient broadband double-sided Fresnel lens for THz range," Optics Letters, vol. 37, no. 12, pp. 2214-2216, 2012.

[11] E. D. Walsby, S. M. Durbin, D. R. S. Cumming, and R. J. Blaikie, "Analysis of silicon terahertz diffractive optics," Current Applied Physics, vol. 4, no. 2-4, pp. 102-105, 2004.

[12] B. S.-Y. Ung, B. Weng, R. Shepherd, D. Abbott, and C. Fumeaux, "Inkjet printed conductive polymer-based beam-splitters for terahertz applications," Optical Materials Express, vol. 3, no. 9, pp. 1242-1249, 2013.

[13] A. Siemion, A. Siemion, M. Makowski et al., "Off-axis metallic diffractive lens for terahertz beams," Optics Letters, vol. 36, no. 11, pp. 1960-1962, 2011.

[14] E. Hérault, J.-L. Coutaz, A. Siemion, A. Siemion, M. Makowski, and M. Sypek, "Prism-like behavior at terahertz frequencies of a 2D metallic grid with a varying periodicity," Journal of Infrared, Millimeter, and Terahertz Waves, vol. 32, no. 4, pp. 403-406, 2011.

[15] D. Han, K. Lee, J. Lim, S. S. Hong, Y. K. Kim, and J. Ahn, "Terahertz lens made out of natural stone," Applied Optics, vol. 52, no. 36, pp. 8670-8675, 2013.

[16] D. Hu, G. Moreno, X. Wang et al., "Dispersion characteristic of ultrathin terahertz planar lenses based on metasurface," Optics Communications, vol. 322, pp. 164-168, 2014.

[17] S. F. Busch, M. Weidenbach, M. Fey, F. Schäfer, T. Probst, and M. Koch, "Optical properties of 3D printable plastics in the $\mathrm{THz}$ regime and their application for 3D printed THz optics," Journal of Infrared, Millimeter, and Terahertz Waves, vol. 35, no. 12, pp. 993-997, 2014.

[18] A. Siemion, J. Suszek, M. Sypek et al., "THz characterization of selected 3D-printing materials," in Proceedings of the 8th $\mathrm{THz}$ Days, Areches-Beaufort, France, 2015.

[19] B. Scherger, M. Scheller, N. Vieweg, S. T. Cundiff, and M. Koch, "Paper terahertz wave plates," Optics Express, vol. 19, no. 25, pp. 24884-24889, 2011.

[20] A. Siemion, A. Siemion, M. Makowski et al., "Diffractive paper lens for terahertz optics," Optics Letters, vol. 37, no. 20, pp. 43204322, 2012.

[21] J. Suszek, M. Sypek, M. Makowski et al., "Evaluation of the shadow effect in terahertz kinoform gratings," Optics Letters, vol. 38, no. 9, pp. 1464-1466, 2013.

[22] P. Lalanne, S. Astilean, P. Chavel, E. Cambril, and H. Launois, "Design and fabrication of blazed binary diffractive elements with sampling periods smaller than the structural cutoff," Journal of the Optical Society of America A, vol. 16, no. 5, pp. 1143-1156, 1999.

[23] D. W. Prather, D. Pustai, and S. Shi, "Performance of multilevel diffractive lenses as a function of f-number," Applied Optics, vol. 40, no. 2, pp. 207-210, 2001.

[24] J. W. Goodman, Introduction to Fourier Optics, McGraw-Hill, New York, NY, USA, 2004.

[25] http://mcortechnologies.com/3d-printers/iris/.

[26] M. Rachoń, K. Wegrzynska, M. Sypek et al., "Efficiency of $\mathrm{THz}$ paper optical elements depending on their type and manufacturing techniques," OSA Technical Digest, 2015.

[27] L. Duvillaret, F. Garet, and J.-L. Coutaz, "A reliable method for extraction of material parameters in terahertz time-domain spectroscopy," IEEE Journal on Selected Topics in Quantum Electronics, vol. 2, no. 3, pp. 739-745, 1996.
[28] A. Siemion, A. Siemion, J. Suszek et al., "THz Beam Shaping Based on Paper Diffractive Optics," IEEE Transactions on Terahertz Science and Technology, vol. 6, no. 4, pp. 568-575, 2016.

[29] J. Suszek, A. Siemion, D. Coquillat et al., "3D printed flat optics and InP heterojunction bipolar transistor based-detector for $\mathrm{THz}$ imaging," in Proceedings of the 40th International Conference on Infrared, Millimeter, and Terahertz Waves, pp. 1-2, 2015.

[30] M. Sypek, "Light propagation in the Fresnel region. New numerical approach," Optics Communications, vol. 116, no. 1-3, pp. 43-48, 1995.

[31] M. Sypek, C. Prokopowicz, and M. Gorecki, "Image multiplying and high-frequency oscillations effects in the Fresnel region light propagation simulation," Optical Engineering, vol. 42, no. 11, pp. 3158-3164, 2003.

[32] B. Scherger, M. Scheller, N. Vieweg, S. T. Cundiff, and M. Koch, "Paper terahertz wave plates," Optics Express, vol. 19, no. 25, pp. 24884-24889, 2011. 

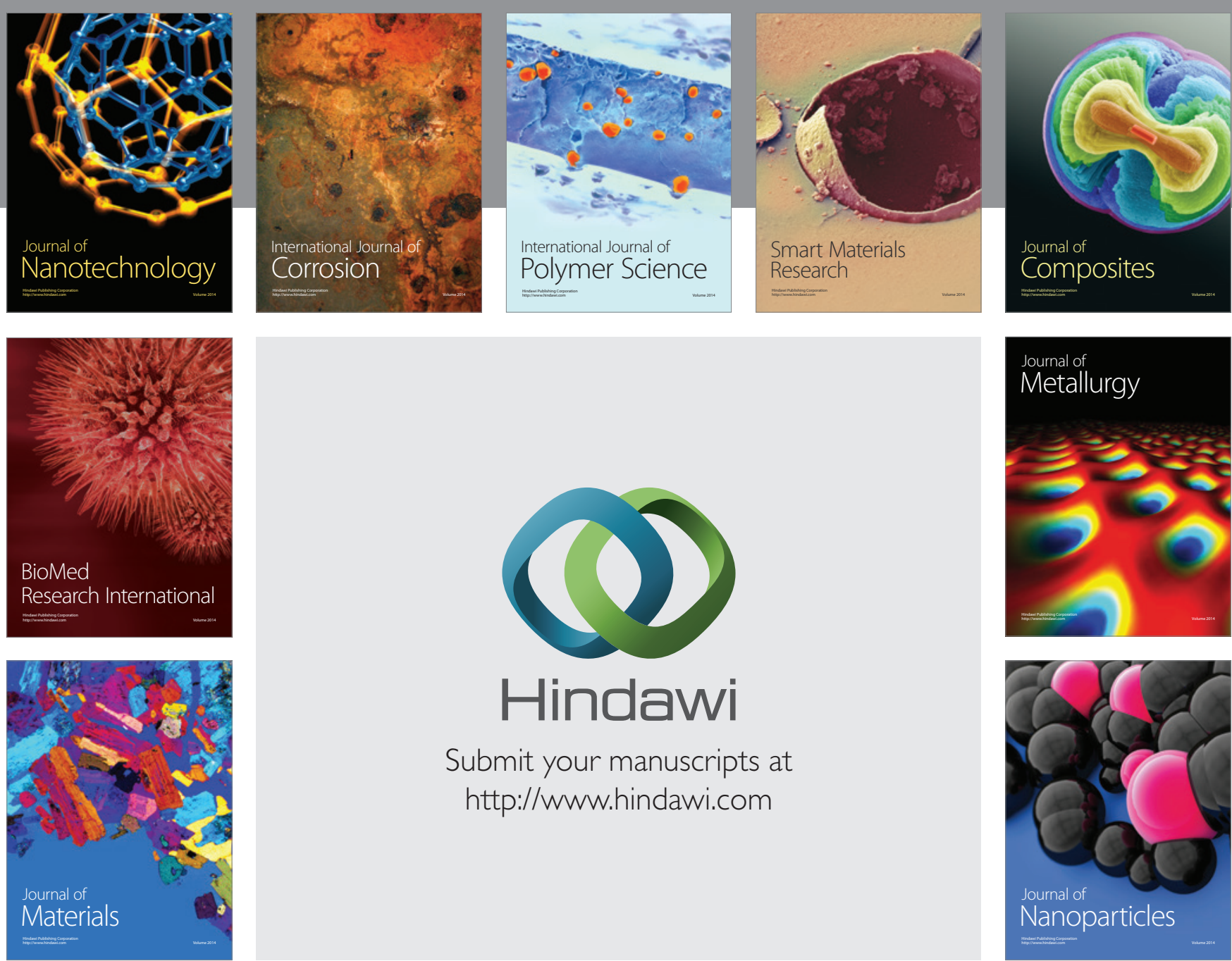

\section{Hindawi}

Submit your manuscripts at

http://www.hindawi.com

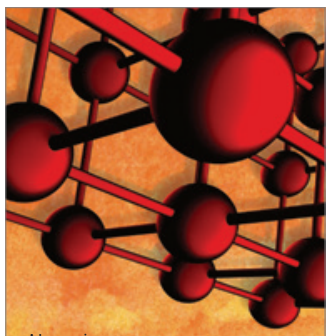

Materials Science and Engineering
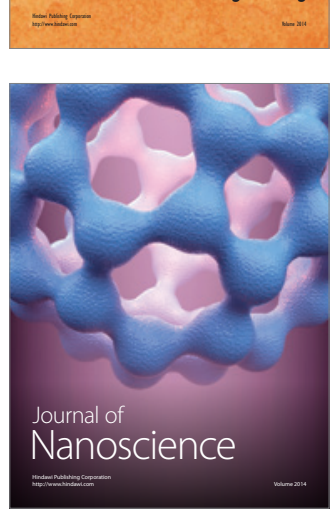
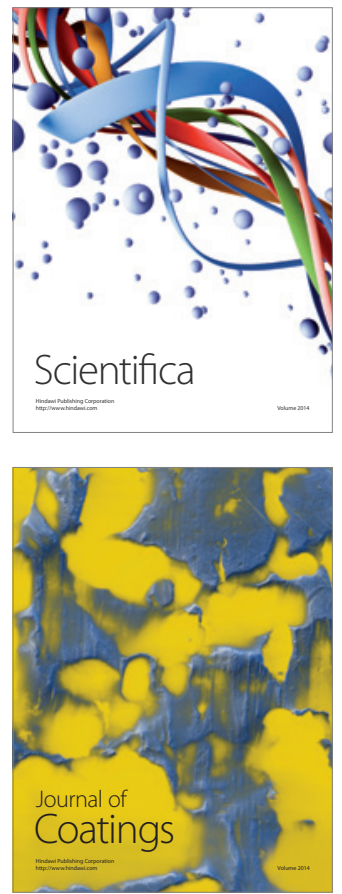
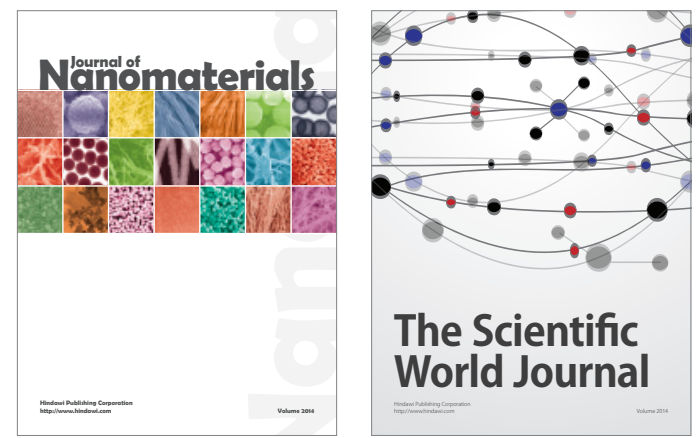

The Scientific World Journal
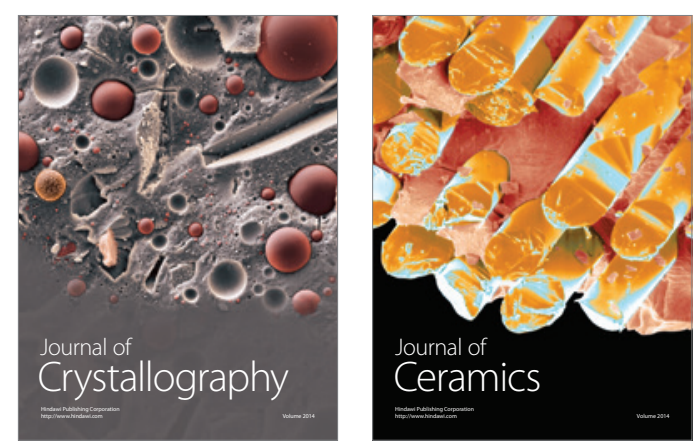
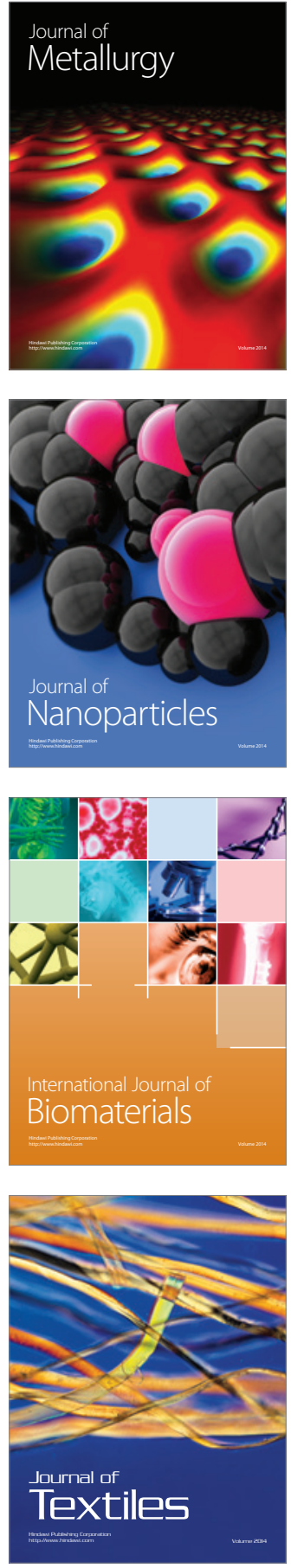